\title{
Synthesis of azacrown sulfonamides via ring closing metathesis and their evaluation in lithium ion selective electrodes
}

\author{
Yehia A. Ibrahim*, Haider Behbehani, Elizabeth John, Nadia M. Shuaib and Adel F. \\ Shoukry
}

Chemistry Department, Faculty of Science, Kuwait University, P.O. Box 5969, Safat 13060, Kuwait

E-mail: yehiaai@kuc01.kuniv.edu.kw

\section{Dedicated to Professor Nouria A. Al-Awadi on the occasion of her $55^{\text {th }}$ birthyear, for her scientific achievements and on the occasion of her appointment as Vice-President for Academic Affairs, Kuwait University}

\begin{abstract}
New diazapolyoxa macrocyclic ditosylates with 17-28-membered rings were synthesized by the application of the RCM technique to suitable $\alpha, \omega$-dienes. These compounds were employed as neutral carriers in $\mathrm{Li}^{+}$-selective electrodes. The electrodes exhibited nearly Nernstian responses with relatively high selectivity for lithium over other inorganic cations.
\end{abstract}

Keywords: Ring closing metathesis, $\alpha, \omega$-dienes, azacrown ethers, crown-sulfonamides, ionselective electrodes, lithium, PVC membrane

\section{Introduction}

Efficient synthetic approaches to macrocyclic heterocyclic compounds have recently been developed using the RCM methodology. ${ }^{1}$ In the present work we applied our previously developed reaction sequence, ${ }^{2-4}$ using the RCM technique, for the macrocyclization step to prepare a number of macrocyclic diazacrown ditosylates with varying ring sizes, in order to investigate their behavior in Li ion-selective electrodes.

Lithium is an important element in biological systems. It has been found to be effective in preventing recurring bouts of depression and elation. However, it is toxic in relatively high concentrations. Therefore, it is important to maintain its concentration in the blood within the range of 0.5-1.5 mM. Lithium is present in human, animal and plant tissues in small amounts. ${ }^{5-8}$ Early lithium ion-selective electrodes were based on amide group type ionophores. ${ }^{9-13}$ Lithium ions were determined in undiluted serum using cyclohexyl diamides based electrodes which had 
a selectivity of 80:1 for lithium over sodium ions. ${ }^{12}$ A series of diamides based on pyridine, furan and dioxanone backbones, which have higher selectivity for lithium over sodium were prepared. ${ }^{13}$ In the present study, coated silver/silver chloride electrodes were prepared based on the following ionophores: 2c, 2d, 4c, 4d, 3c, 3d, 5c, 5d, 6d and 7d as $\mathrm{Li}^{+}$-neutral carriers (Scheme 1).

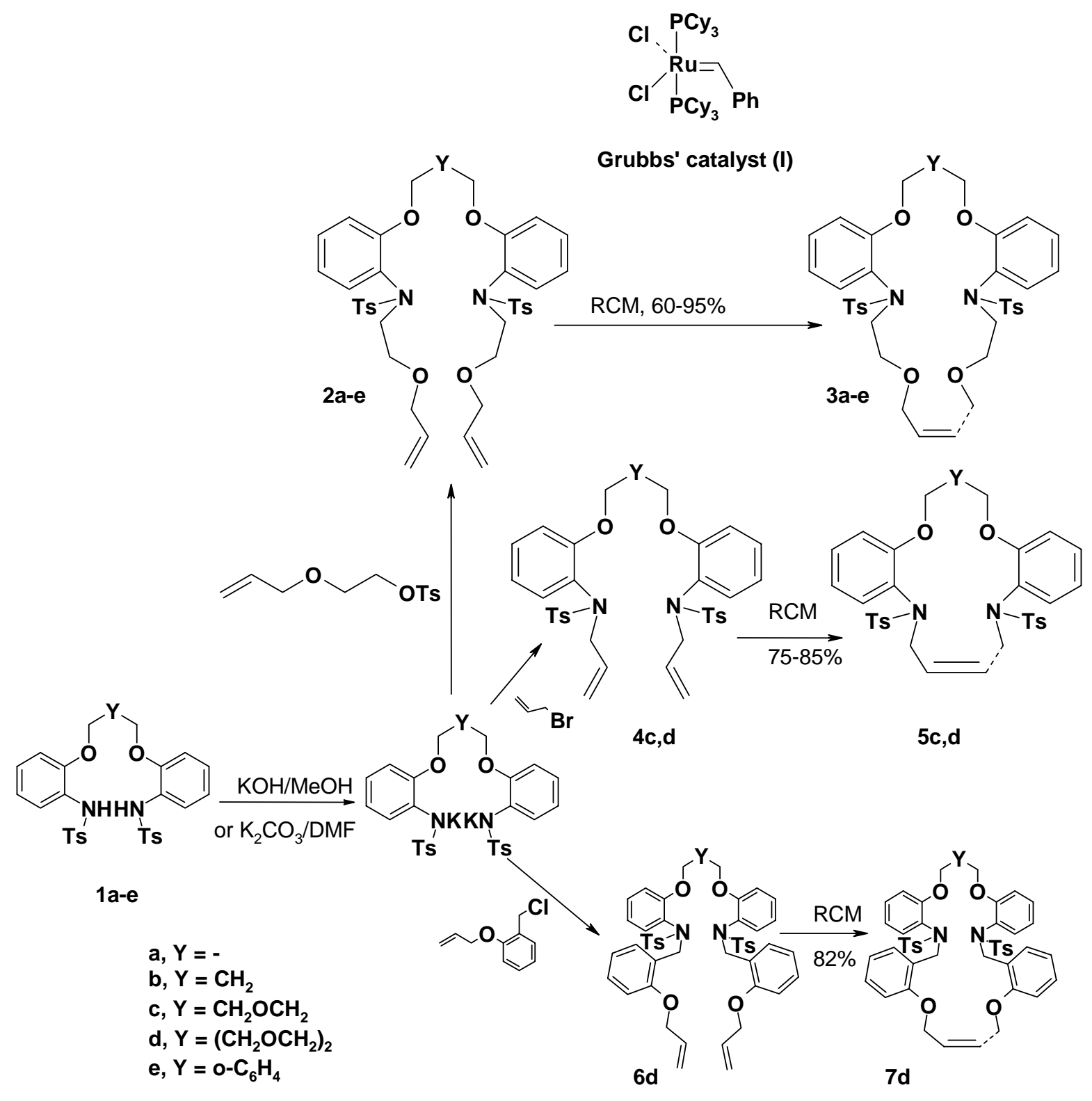

RCM conditions: Grubbs' catalyst I (1.5-5mol\%), DCM, Reflux 2 h

\section{Scheme 1}




\section{Results and Discussion}

Scheme 1 illustrates our synthetic routes starting from the appropriate readily available bistosylamides 1a-e which were converted via their potassium salts into the corresponding 1, $\omega$ dienes 2, 4, 6 upon treatment with allyloxyethyl tosylate, allyl bromide or $o$-allyloxybenzyl chloride. RCM of these dienes proceeded under mild condition using 1-5 mol\% of Grubbs' catalyst $\mathbf{I}$ in $\mathrm{CH}_{2} \mathrm{Cl}_{2}$ to give excellent yields of the corresponding macrocyclic products $\mathbf{3}, \mathbf{5}$ and 7 respectively as an $E / Z$ mixture in a ratio determined from their ${ }^{1} \mathrm{H}$ NMR spectra as shown in Table 1 ( $c f$. Experimental section for $E$ and $Z$ isomer assignments).

Table 1. Catalyst $\%$, yields and $E / Z$ ratios of macrocycles $3 a-e, 5 b, d$ and $7 d$

\begin{tabular}{ccccc}
\hline Entry & Substrate & Mol\% catalyst/substrate & Yield (\%) & Product $\boldsymbol{E}: \mathbf{Z}$ ratio \\
\hline 1 & 2a & 1.5 & 80 & 3a $1.8: 1$ \\
2 & 2b & 1 & 86 & $3 \mathbf{b} 2: 1$ \\
3 & 2c & 1 & 80 & 3c $2: 1$ \\
4 & 2d & 5 & 60 & 3d $2.2: 1$ \\
5 & 2e & 5 & 95 & 3e $3: 1$ \\
6 & 4c & 1 & 75 & $\mathbf{5 b} 1.8: 1$ \\
7 & 4d & 3 & 85 & $\mathbf{5 d ~} 1: 1$ \\
8 & 6d & 5 & 82 & $\mathbf{7 d ~} 1.7: 1$ \\
\hline
\end{tabular}

The prepared compounds were investigated as neutral carriers for $\mathrm{Li}^{+}$ion-selective electrodes. The selectivity coefficients of the electrodes towards different cationic species $\left(\mathrm{M}^{\mathrm{n}+}\right)$ were determined by the matched potential method (MPM). ${ }^{14}$ According to this method the selectivity coefficient was measured by determining the activity ratio of the primary ion $\left(\mathrm{Li}^{+}\right)$ and the interfering ion $\left(\mathrm{M}^{\mathrm{n}+}\right)$ that gives the same potential change when added to a reference $\mathrm{Li}^{+}$ solution. Thus, we measured the change in potential upon changing the lithium ion activity, then the interfering ion was added to an identical reference solution until the same potential change was obtained.

The matched potential method was applied to calculate the selectivity coefficients for the electrodes where $\mathrm{M}^{\mathrm{n}+}$ is $\mathrm{K}^{+}, \mathrm{Na}^{+}, \mathrm{Ca}^{2+}, \mathrm{Ni}^{2+}, \mathrm{Cu}^{2+}, \mathrm{Cd}^{2+}, \mathrm{Co}^{2+}, \mathrm{Mn}^{2+}, \mathrm{Zn}^{2+}, \mathrm{Mg}^{2+}$ and $\mathrm{Pb}^{2+}$. The results revealed that all electrodes showed highest values of selectivity coefficients for lithium ion $\left(\mathrm{Li}^{+}\right)$towards $\left(\mathrm{Cd}^{2+}\right)$ followed by $\left(\mathrm{Na}^{+}\right)$in the case of electrodes $2 \mathrm{c}, 3 \mathbf{c}, 3 \mathbf{d}, \mathbf{4 d}, \mathbf{6}$ and 7. As for $\mathrm{Cd}^{2+}$, the order of decrease of interference was $\mathbf{3 c}=\mathbf{7}>\mathbf{3 d}=\mathbf{4 c}>\mathbf{2 c}>\mathbf{4 d}>\mathbf{6 d}$. The higher selectivity coefficients (less selectivity) of compounds $3 \mathbf{c}$ and $\mathbf{7}$ than the other compounds with respect to $\mathrm{Cd}^{2+}$ is most probably due to the ring closing system. 
Table 2. Logarithmic values of selectivity coefficients for various lithium ion $\left(\mathrm{Li}^{+}\right)$selective electrodes

\begin{tabular}{cccccccccccc}
\hline Electrode & $\mathrm{K}^{+}$ & $\mathrm{Na}^{+}$ & $\mathrm{Ca}^{2+}$ & $\mathrm{Ni}^{2+}$ & $\mathrm{Cu}^{2+}$ & $\mathrm{Cd}^{2+}$ & $\mathrm{Co}^{2+}$ & $\mathrm{Mn}^{2+}$ & $\mathrm{Zn}^{2+}$ & $\mathrm{Mg}^{2+}$ & $\mathrm{Pb}^{2+}$ \\
\hline No ionophore & -1.6 & -1.08 & -1.7 & -2.2 & -1.32 & -0.6 & -2.5 & -2.06 & -1.64 & -2.62 & -1.53 \\
2c & -1.55 & -1 & -1.77 & -2.41 & -1.48 & -0.81 & -2.85 & -2.64 & -1.82 & -2.62 & -1.44 \\
2d & -1.65 & -1.4 & -1.9 & -2.9 & -2.6 & -1.08 & -2.3 & -2.5 & -3.17 & -2.2 & -1.4 \\
3c & -1.7 & -1.14 & -1.68 & -2.18 & -1.41 & -0.79 & -2.66 & -2.52 & -1.78 & -2.79 & -1.64 \\
3d & -1.63 & -1.07 & -1.44 & -2.16 & -1.49 & -0.8 & -2.71 & -2.2 & -1.9 & -2.71 & -1.38 \\
$\mathbf{4 c}$ & -1.7 & -0.92 & -1.77 & -2.03 & -1.37 & -0.8 & -2.28 & -2.38 & -1.72 & -2.07 & -1.25 \\
$\mathbf{4 d}$ & -1.72 & -1 & -2.08 & -2.26 & -1.54 & -0.9 & -2.51 & -2.37 & -1.79 & -3.15 & -1.41 \\
$\mathbf{5 c}$ & -1.85 & -1.24 & -2.33 & -2.14 & -1.49 & -1.02 & -2.7 & -2.66 & -1.95 & -3.2 & -1.34 \\
$\mathbf{5 d}$ & -1.77 & -1.32 & -2 & -2.36 & -1.59 & -1.06 & -2.94 & -2.66 & -2.33 & -2.94 & -1.47 \\
$\mathbf{6 d}$ & -1.69 & -1.16 & -1.93 & -2.18 & -1.54 & -0.91 & -2.87 & -2.69 & -1.85 & -2.97 & -1.4 \\
7d & -1.93 & -1.28 & -2.19 & -2.35 & -1.58 & -0.79 & -2.83 & -2.61 & -1.88 & -2.55 & -1.2 \\
\hline
\end{tabular}

In general, it is clear from the results given in Table 2 that the investigated compounds exhibit reasonable selectivity towards $\mathrm{Li}^{+}$cations. It is also noticeable that other univalent metal cations $\left(\mathrm{Na}^{+}\right.$and $\left.\mathrm{K}^{+}\right)$interfere more than divalent species (with the exception of $\mathrm{Cd}^{2+}$ ).

\section{Experimental Section}

All melting points are uncorrected. IR spectra were recorded in $\mathrm{KBr}$ disks using a Perkin Elmer System 2000 FT-IR spectrophotometer. ${ }^{1} \mathrm{H}$ and ${ }^{13} \mathrm{C}$ NMR spectra were recorded on a Bruker DPX 400, $400 \mathrm{MHz}$ super-conducting NMR spectrometer. Mass spectra were measured on a VG Auto-spec-Q (high resolution, high performance, tri-sector GC/MS/MS) and with LCMS using Agilent 1100 series LC/MSD with an API-ES/APCI ionization mode. Microanalyses were performed on a LECO CH NS-932 Elemental Analyzer. The starting bis tosylamides 1a-e were prepared by reacting the appropriate bisamines, readily synthesizable as reported, ${ }^{15}$ with tosyl chloride in pyridine. These bis tosyl derivatives 1a-e are reported but without complete identification. ${ }^{16-18}$ Poly(vinylchloride) of high molecular weight, tetrahydrofuran (THF), and potassium tetrakis-(4-chlorophenyl)borate (KTpClPB), tris(2-ethylhexyl)phosphate (TEHP), were obtained from Aldrich. Solutions of metal salts in the chloride form (Aldrich) were prepared in deionized water.

\section{Potential measurements}

All the measurements were carried out using a $713 \mathrm{pH}-\mathrm{mv}$ meter (Metrohm, Swiss), and a Titrator 665 Dosimat (Metrohm, Swiss). The electrochemical system was as follows:

$\mathrm{Ag}|\mathrm{AgCl}| \mathrm{KCl}$ (sat'd)|3.0 $\mathrm{M} \mathrm{KNO}_{3} \mid$ sample solution|membrane $|\mathrm{AgCl}| \mathrm{Ag}$ 


\section{Electrode preparation}

All electrodes were prepared as previously described. ${ }^{19}$ Pure silver rods of $10 \mathrm{~cm}$ length and 5.0 $\mathrm{mm}$ diameter, purchased from Goodfellow Cambridge LTD, England, were insulated by tight polyethylene tubes leaving $2 \mathrm{~cm}$ at one end for connection and $1 \mathrm{~mm}$ at the other end to be coated anodically with $\mathrm{AgCl}$. Membrane solution was prepared by dissolving $18.00 \mathrm{mg}$ of the ionophore, $139 \mathrm{mg}$ of PVC, $139 \mathrm{mg}$ of plasticizer (tris-2-ethylhexylphosphate, TEHP), and 1.42 $\mathrm{mg}$ potassium tetrakis(4-chlorophenyl) borate (KTpClPB) in $500 \mu \mathrm{L}$ tetrahydrofuran (THF).

\section{Electrode Construction}

Two $25 \mu \mathrm{L}$ aliquots of the membrane solution were carefully deposited on the sliver-silver chloride electrode surface. The second aliquot was deposited after the first aliquot had almost dried. The tip was left to stand for $24 \mathrm{~h}$ to allow the THF solvent to evaporate, and then it was soaked in $0.1 \mathrm{M}$ of lithium chloride solution $(\mathrm{LiCl})$ for $24 \mathrm{~h}$ before use.

\section{Compounds 1a-e}

General Procedure. To an ice cold solution of the appropriate bisamine dihydrochloride (10 $\mathrm{mmol})$ in dry pyridine, was added $p$-toluenesulfonyl chloride $(21 \mathrm{mmol})$. The mixture was stirred at $0{ }^{\circ} \mathrm{C}$ for $4 \mathrm{~h}$ and then kept overnight in the fridge. The precipitate obtained after addition of cold water was collected and crystallized from $\mathrm{EtOH} / \mathrm{CHCl}_{3}$.

Compound 1a. Yield 78\%, mp 189-190 ${ }^{\circ} \mathrm{C}$, LCMS; m/z 553 (M + 1). IR: 3434, 3273, 1599, $1501,1401,1342,1246,1165,1115,1091,918,746,673,564 .{ }^{1} \mathrm{H}$ NMR $\left(\mathrm{CDCl}_{3}\right): \delta 7.62(\mathrm{~d}, 4 \mathrm{H}$, $J$ 8.2), 7.55 (dd, $2 \mathrm{H}, J$ 7.9, 1.2), 7.15 (d, 4H, J 8.2), 7.12 ( dt, 2H, J 7.4, 1.2), 6.99 (t, 2H, J 7.6), $6.96(\mathrm{~s}, 2 \mathrm{H}, \mathrm{NH}), 6.80\left(\mathrm{~d}, 2 \mathrm{H}, J\right.$ 7.9), $4.07\left(\mathrm{~s}, 4 \mathrm{H}, \mathrm{CH}_{2}\right), 2.32\left(\mathrm{~s}, 6 \mathrm{H}, \mathrm{CH}_{3}\right)$. Anal. calcd. for $\mathrm{C}_{28} \mathrm{H}_{28} \mathrm{~N}_{2} \mathrm{O}_{6} \mathrm{~S}_{2}$ (552.6): C 60.85; H 5.11; N 5.07\%; S 11.6. Found: C 60.67; H 4.89; N 5.28; $\mathrm{S}$ $11.26 \%$.

Compound 1b. Yield $68 \%$, mp $249-250{ }^{\circ} \mathrm{C}$ (lit. ${ }^{16}$, mp 228-230 ${ }^{\circ} \mathrm{C}$ ), LCMS; $m / z 567(\mathrm{M}+1)$. IR: $3277,2950,2890,1598,1501,1474,1400,1336,1290,1252,1163,1112,1094,1052,919,814$, 753, 672, 564, 543. ${ }^{1} \mathrm{H}$ NMR $\left(\mathrm{CDCl}_{3}\right): \delta 7.61(\mathrm{~d}, 4 \mathrm{H}, J$ 8.1), $7.55(\mathrm{~d}, 2 \mathrm{H}, J$ 8.2), 7.08 (d, $6 \mathrm{H}, J$ 7.3), $6.96\left(\mathrm{t}, 2 \mathrm{H}, J\right.$ 7), $6.93(\mathrm{~s}, 2 \mathrm{H}, \mathrm{NH}), 6.77$ (d, 2H, J 8.1), 3.94 ( t, $4 \mathrm{H}, J$ 5.4, $\left.\mathrm{CH}_{2}\right), 2.29(\mathrm{~s}, 6 \mathrm{H}$, $\mathrm{CH}_{3}$ ), 2.09 (q, $2 \mathrm{H}, \mathrm{J}$ 5.7, $\mathrm{CH}_{2}$ ). Anal. calcd. for $\mathrm{C}_{29} \mathrm{H}_{30} \mathrm{~N}_{2} \mathrm{O}_{6} \mathrm{~S}_{2}$ (566.7): C 61.47; H 5.34; N 4.94; S 11.32\%. Found: C 61.31; H 5.76; N 5.48; S 11.39\%.

Compound 1c. Yield (89\%), mp 163-164 ${ }^{\circ} \mathrm{C}$, LCMS; m/z 597 (M + 1). IR: 3270, 3061, 2934, 2884, 1599, 1501, 1456, 1408, 1393, 1337, 1289, 1257, 1163, 1139, 1112, 1091, 1057, 932, 815, $750,680,565,543 .{ }^{1} \mathrm{H}$ NMR $\left(\mathrm{CDCl}_{3}\right): \delta 7.65(\mathrm{~d}, 4 \mathrm{H}, J$ 7.1), 7.56-7.52 (m, 4H), $7.09(\mathrm{~d}, 4 \mathrm{H}, J$ 7.2), 7.04-7.00 (m, 2H), 6.95-6.96 (m, 2H), 6.86 (d, 2H, J 7.7), 4.09 (s, 4H), 3.82 (s, 4H), 2.26 (s, $6 \mathrm{H}, \mathrm{CH}_{3}$ ). Anal. calcd. for $\mathrm{C}_{28} \mathrm{H}_{28} \mathrm{~N}_{2} \mathrm{O}_{6} \mathrm{~S}_{2}$ (596.7): $\mathrm{C} 60.39 ; \mathrm{H}$ 5.41; $\mathrm{N} 4.69 ; \mathrm{S} 10.75 \%$. Found: $\mathrm{C}$ 60.05; H 5.86; N 5.05; S 10.60\%.

Compound 1d. Yield (60\%), mp $119^{\circ} \mathrm{C}$. LCMS; $\mathrm{m} / \mathrm{z} 641$ (M + 1). IR: 3251, 3063, 2926, 2877 , 1599, 1498, 1455, 1410, 1337, 1290, 1262, 1162, 1131, 1114, 1094, 1042, 936, 909, 746, 681, 
568. ${ }^{1} \mathrm{H}$ NMR $\left(\mathrm{CDCl}_{3}\right): \delta 7.68(\mathrm{~d}, 4 \mathrm{H}, J$ 8.2), 7.57 (s, 2H, NH), 7.54 (dd, 2H, J 7.9, 1.5), 7.16 (d, $4 \mathrm{H}, J$ 8.1), 7.00 (dt, 2H, J 7.7, 1.6), 6.93 (dt, 2H, J 7.6, 1), 6.80 (dd, 2H, J 8, 1.1), 3.99-3.96 (m, $4 \mathrm{H}), 3.84(\mathrm{~s}, 4 \mathrm{H}), 3.76-3.75(\mathrm{~m}, 4 \mathrm{H}), 2.33\left(\mathrm{~s}, 6 \mathrm{H}, \mathrm{CH}_{3}\right)$. Anal. calcd. for $\mathrm{C}_{32} \mathrm{H}_{36} \mathrm{~N}_{2} \mathrm{O}_{8} \mathrm{~S}_{2}(640.8)$ : C 59.98; H 5.66; N 4.37; S 10.01\%. Found: C 59.83; H 6.11; N 4.54; S 10.25\%.

Compound 1e. Yield (50\%), mp 146-147 ${ }^{\circ} \mathrm{C}$, LCMS; m/z 629 (M + 1). IR: 3557, 3258, 2922 , $1598,1498,1405,1335,1252,1163,1112,1092,1000,910,812,751,672,570,539 .{ }^{1} \mathrm{H}$ NMR $\left(\mathrm{CDCl}_{3}\right): \delta 7.61(\mathrm{~d}, 4 \mathrm{H}, J$ 8.2), $7.55(\mathrm{dd}, 2 \mathrm{H}, J 7.9,1.6), 7.42-7.40(\mathrm{~m}, 2 \mathrm{H}), 7.22-7.20(\mathrm{~m}, 2 \mathrm{H})$, $7.16(\mathrm{~d}, 4 \mathrm{H}, J$ 8.1), 7.04-7.00 (m, 4H), 6.95 (dt, 2H, J 7.6, 1.1), 6.74 (dd, 2H, J 7.5, 0.9), 4.89 (s, $\left.4 \mathrm{H}, \mathrm{CH}_{2}\right), 2.36\left(\mathrm{~s}, 6 \mathrm{H}, \mathrm{CH}_{3}\right)$. Anal. calcd. for $\mathrm{C}_{34} \mathrm{H}_{32} \mathrm{~N}_{2} \mathrm{O}_{6} \mathrm{~S}_{2}(628.7)$ : C 64.95; H 5.13; N 4.46; $\mathrm{S}$ 10.20\%. Found: C 64.75; H 5.49; N 4.80; S 9.58\%.

\section{Synthesis of compounds 2a-e, 4c, 4d and 6}

General procedure A. A mixture of the appropriate bistosylamide 1a-e (10 mmol), allyloxyethyl tosylate, allyl bromide or $O$-allyloxybenzyl chloride $(20 \mathrm{mmol})$ and anhydrous $\mathrm{K}_{2} \mathrm{CO}_{3}(5.52 \mathrm{~g}, 40 \mathrm{mmol})$ was stirred in dry DMF $(5 \mathrm{~mL})$ at $100{ }^{\circ} \mathrm{C}$ for $16 \mathrm{~h}$. Crushed ice was added to the mixture and the precipitate was collected, washed with $\mathrm{KOH}$ solution (10\%) and with water several times and then extracted with DCM, dried over $\mathrm{Na}_{2} \mathrm{SO}_{4}$ and evaporated to give 2a-e, 4c,d and 6 respectively.

General procedure B. To a solution of $\mathrm{KOH}(10 \mathrm{mmol})$ in methanol $(15 \mathrm{~mL})$ was added each of 1a-e $(10 \mathrm{mmol})$. The mixture was then stirred at room temperature for $15 \mathrm{~min}$ and the solvent was then removed in vacuo. To the remaining potassium salt was added DMF (2 mL) and allyloxyethyl tosylate, allyl bromide or $o$-allyloxybenzyl chloride $(20 \mathrm{mmol})$. The reaction mixture was then heated under reflux for $5 \mathrm{~min}$. The mixture was cooled, diluted with water (20 $\mathrm{mL}$ ) and extracted with DCM, washed with cold water several times, dried over anhydrous $\mathrm{Na}_{2} \mathrm{SO}_{4}$ and evaporated to give $2 \mathbf{a}-\mathbf{e}, \mathbf{4 c}, \mathbf{d}$ and $\mathbf{6}$ respectively.

Compound 2a. Yield 58\% (A), 31\% (B); colorless crystals (EtOH), mp 125-126 ${ }^{\circ} \mathrm{C}, \mathrm{R}_{\mathrm{f}}=0.8$ (DCM/pet. ether 40-60/EtOAc 6:4:2). LCMS; m/z 721 (M + 1). IR: 3071, 2889, 2857, 1596, 1498, 1479, 1456, 1345, 1285, 1257, 1164, 1102, 1067, 1049, 916, 818, 799, 750, 712, 696, 655. ${ }^{1} \mathrm{H}$ NMR $\left(\mathrm{CDCl}_{3}\right): \delta 7.59(\mathrm{~d}, 4 \mathrm{H}, J$ 8.1), $7.35(\mathrm{dt}, 2 \mathrm{H}, J$ 8.2, 1.5), $7.26(\mathrm{dd}, 4 \mathrm{H}, J$ 7.8, 1.5), 7.22 (d, 2H, J 8.1), 6.98 (dt, 2H, J 7.7, 0.9), 6.78 (d, 2H, J 7.6), $5.78(\mathrm{~m}, 2 \mathrm{H},-\mathrm{CH}=), 5.17$ (dd, 2H, 17.3, 1.5, $\left.\mathrm{CH}_{2}=\mathrm{CH}\right), 5.12\left(\mathrm{dd}, 2 \mathrm{H}, J 10.4,1.2, \mathrm{CH}_{2} \mathrm{CH}=\right), 3.87\left(\mathrm{~d}, 4 \mathrm{H}, J 5.5, \mathrm{OCH}_{2} \mathrm{CH}=\right), 3.73$ $\left(\mathrm{m}, 4 \mathrm{H}, \mathrm{CH}_{2}\right), 3.59\left(\mathrm{~s}, 4 \mathrm{H}, \mathrm{CH}_{2}\right), 3.49\left(\mathrm{t}, 4 \mathrm{H}, J 6.2, \mathrm{CH}_{2}\right), 2.36\left(\mathrm{~s}, 6 \mathrm{H}, \mathrm{CH}_{3}\right) .{ }^{13} \mathrm{C} \mathrm{NMR}\left(\mathrm{CDCl}_{3}\right)$ : $\delta 155.4,142.8,137.9,134.5,133.2,129.8,129.1,127.6,121.2,116.9,112.6,71.8,68.3,68.1$, 65.7, 49.3, 21.4\%. Anal. calcd. for $\mathrm{C}_{38} \mathrm{H}_{44} \mathrm{~N}_{2} \mathrm{O}_{8} \mathrm{~S}_{2}$ (720.9): C 63.31; H 6.15; N 3.89; S 8.90. Found: C 63.28; H 6.00; N 4.29; S 8.72\%.

Compound 2b. Yield 93\% (A), 73\% (B); colorless crystals (EtOH), mp 136-137 ${ }^{\circ} \mathrm{C}, \mathrm{R}_{\mathrm{f}}=0.9$ (DCM/pet. ether 40-60/EtOAc 6:4:2). LCMS; m/z 735 (M + 1). IR: 3441, 2942, 2884, 1596, 1496, 1454, 1344, 1280, 1250, 1163, 1117, 1089, 1064, 916, 820, 749. ${ }^{1} \mathrm{H} \mathrm{NMR}\left(\mathrm{CDCl}_{3}\right): \delta 7.61$ 
(d, 4H, J 8.1), 7.29 (dt, 2H, J 8, 1.5), 7.24 (d, 4H, J 8.1), $7.16(\mathrm{dd}, 2 \mathrm{H}, J$ 7.7, 1.4), 6.90 (m, 4H), $5.78(\mathrm{~m}, 2 \mathrm{H}, \mathrm{CH}=), 5.15\left(\mathrm{dd}, 2 \mathrm{H}, J 17.3,1.3, \mathrm{CH}_{2}=\mathrm{CH}\right), 5.11\left(\mathrm{~d}, 2 \mathrm{H}, J 10.8, \mathrm{CH}_{2}=\mathrm{CH}\right), 3.85(\mathrm{~d}$, $4 \mathrm{H}, J$ 5.4, $\left.\mathrm{OCH}_{2} \mathrm{CH}=\right)$, 3.90-3.65 (br, 8H), 3.48 (t, 4H, J 6.3), 2.41 (s, 6H, $\mathrm{CH}_{3}$ ), 1.61 (br, 2H) . ${ }^{13} \mathrm{C} \mathrm{NMR}\left(\mathrm{CDCl}_{3}\right): \delta 155.9,142.8,137.7,134.5,132.5,129.5,129.1,127.6,127.1,120.6,116.9$, 112.6, 71.8, 68.3, 64.4, 49.3, 28.5, 21.5. Anal. calcd. for $\mathrm{C}_{39} \mathrm{H}_{46} \mathrm{~N}_{2} \mathrm{O}_{8} \mathrm{~S}_{2}$ (734.9): C 63.74; $\mathrm{H}$ 6.31; N 3.81; S 8.73\%. Found: C 63.44; H 6.72; N 4.02; S 8.85\%.

Compound 2c. Yield 50\% (A), 40\% (B); colorless crystals (EtOAc/pet. ether 40-60), mp 87-88 ${ }^{\circ} \mathrm{C}, \mathrm{R}_{\mathrm{f}}=0.4$ (DCM/pet. ether 40-60/EtOAc 6:4:2). LCMS; m/z 765 (M+1). IR: 3068, 2928, 2868, $1597,1496,1453,1337,1284,1158,1139,1118,1086,927,809,757,717,658 .{ }^{1} \mathrm{H}$ NMR $\left(\mathrm{CDCl}_{3}\right): \delta 7.61(\mathrm{~d}, 4 \mathrm{H}, J$ 8), $7.24(\mathrm{~m}, 8 \mathrm{H}), 6.94(\mathrm{t}, 2 \mathrm{H}, J$ 7.6), $6.84(\mathrm{~d}, 2 \mathrm{H}, J$ 8.2), $5.80(\mathrm{~m}, 2 \mathrm{H}$, $\mathrm{CH}=), 5.17\left(\mathrm{~d}, 2 \mathrm{H}, J 17.2, \mathrm{CH}_{2}=\mathrm{CH}\right), 5.11\left(\mathrm{~d}, 2 \mathrm{H}, J 10.4, \mathrm{CH}_{2}=\mathrm{CH}\right), 3.88\left(\mathrm{~d}, 4 \mathrm{H}, J\right.$ 5.6, $\left.\mathrm{CH}_{2}\right)$, $3.70-3.87(\mathrm{~m}, 8 \mathrm{H}), 3.50\left(\mathrm{t}, 4 \mathrm{H}, J 6.4, \mathrm{CH}_{2}\right), 3.45\left(\mathrm{t}, 4 \mathrm{H}, J 4.5, \mathrm{CH}_{2}\right), 2.42\left(\mathrm{~s}, 6 \mathrm{H}, \mathrm{CH}_{3}\right) .{ }^{13} \mathrm{C}$ NMR $\left(\mathrm{CDCl}_{3}\right): \delta 155.7,142.8,137.8,134.5,132.9,129.7,129.1,127.9,127.7,127.2,120.8$, 116.8, 112.7, 71.8, 69.2, 68.3, 67.2, 49.2, 21.5. Anal. calcd. for $\mathrm{C}_{40} \mathrm{H}_{48} \mathrm{~N}_{2} \mathrm{O}_{9} \mathrm{~S}_{2}$ (764.9): C 62.81; H 6.32; N 3.66; S 8.38\%. Found: C 62.68; H 6.08; N 4.15; S 8.09\%.

Compound 2d. Yield 71\% (A), 60\% (B); colorless oil, purified by column chromatography $\left(\mathrm{DCM} /\right.$ pet. ether 40-60), $\mathrm{R}_{\mathrm{f}}=0.6$ (EtOAc/pet. ether 40-60, 2:1). LCMS; m/z 809 (M+1). IR: 3068, 3015, 2925, 2871, 1597, 1496, 1451, 1345, 1287, 1254, 1163, 1118, 1092, 1073, 929, 816, 755, 711, 657. ${ }^{1} \mathrm{H}$ NMR $\left(\mathrm{CDCl}_{3}\right): \delta 7.60(\mathrm{~d}, 4 \mathrm{H}, J 8.1), 7.27(\mathrm{~m}, 4 \mathrm{H}), 7.23(\mathrm{~d}, 4 \mathrm{H}, J 8.1), 6.94(\mathrm{t}$, $2 \mathrm{H}, J$ 7.5), $6.82(\mathrm{~m}, 2 \mathrm{H}), 5.80\left(\mathrm{~m}, 2 \mathrm{H}, \mathrm{CH}_{2}=\right), 5.18\left(\mathrm{dd}, 2 \mathrm{H}, J 17.4,1.3, \mathrm{CH}_{2}=\mathrm{CH}\right), 5.12(\mathrm{~d}, 2 \mathrm{H}, J$ 10.3, $\left.\mathrm{CH}_{2}=\mathrm{CH}\right), 3.89\left(\mathrm{~d}, 4 \mathrm{H}, J\right.$ 5.5, $\left.\mathrm{OCH}_{2} \mathrm{CH}=\right), 3.83(\mathrm{br}, 8 \mathrm{H}), 3.59$ (s, 4H), 3.52 (t, 4H, J 6.3), $3.43(\mathrm{t}, 4 \mathrm{H}, J 4.6), 2.41\left(\mathrm{~s}, 6 \mathrm{H}, \mathrm{CH}_{3}\right) .{ }^{13} \mathrm{C} \mathrm{NMR}\left(\mathrm{CDCl}_{3}\right): \delta 155.5,142.7,137.7,134.5,133.1$, 129.7, 129.1, 127.6 (2C), 120.8, 116.9, 112.4, 71.8, 70.7, 69.0, 68.4, 67.0, 49.1, 21.5. Anal. calcd. for $\mathrm{C}_{42} \mathrm{H}_{52} \mathrm{~N}_{2} \mathrm{O}_{10} \mathrm{~S}_{2}$ (809.02): C 62.36; H 6.48; N 3.46; S 7.93\%. Found: C 62.08; H 6.44; N 3.87; S 7.70\%.

Compound 2e. Yield 57\% (A), 20\% (B); colorless oil, purified by column chromatography (DCM/pet. ether 40-60/EtOAc), $\mathrm{R}_{\mathrm{f}}=0.9$ (DCM/pet. ether 40-60/EtOAc 6:4:2). LCMS; m/z 797 $(\mathrm{M}+1)$. IR: 3068, 3028, 2925, 2865, 1596, 1497, 1344, 1163, 1091, 755, 656, 579. ${ }^{1} \mathrm{H}$ NMR $\left(\mathrm{CDCl}_{3}\right): \delta 7.58(\mathrm{~d}, 4 \mathrm{H}, J$ 8), $7.31(\mathrm{~m}, 4 \mathrm{H}), 7.22(\mathrm{~m}, 4 \mathrm{H}), 7.09(\mathrm{~d}, 4 \mathrm{H}, J$ 7.8), $7.03(\mathrm{~d}, 2 \mathrm{H}, J$ 8.2), $6.95(\mathrm{t}, 2 \mathrm{H}, J 7.5), 5.78(\mathrm{~m}, 2 \mathrm{H}), 5.14\left(\mathrm{~d}, 2 \mathrm{H}, J 18.2, \mathrm{CH}_{2}=\mathrm{CH}\right), 5.10\left(\mathrm{~d}, 2 \mathrm{H}, J 11.4, \mathrm{CH}_{2}=\mathrm{CH}\right)$, 4.91 (br s, 4H), 3.84 (d, 4H, J 5.1, $\left.\mathrm{OCH}_{2} \mathrm{CH}=\right), 3.80$ (br, 4H), 3.51 (t, 4H, J 6.1, $\mathrm{CH}_{2}$ ), 2.31 (s, $\left.6 \mathrm{H}, \mathrm{CH}_{3}\right) .{ }^{13} \mathrm{C} \mathrm{NMR}\left(\mathrm{CDCl}_{3}\right): \delta 155.7,142.7,137.5,134.4,134.0,132.5,129.8,129.1,128.2$, 128.1, 127.4, 127.3, 120.9, 116.8, 113.1, 71.7, 68.2, 67.6, 49.4, 22.4. Anal. calcd. for $\mathrm{C}_{44} \mathrm{H}_{48} \mathrm{~N}_{2} \mathrm{O}_{8} \mathrm{~S}_{2}$ (797.0): C 66.31; H 6.07; N 3.51; S 8.05\%. Found: C 66.08; H 6.04; N 3.80; S $7.88 \%$.

Compound 4c. Yield 63\% (A), 96\% (B); colorless crystals $(\mathrm{EtOH}), \mathrm{mp} 125-126{ }^{\circ} \mathrm{C}, \mathrm{R}_{\mathrm{f}}=0.9$ (DCM/pet. ether 40-60/EtOAc 6:4:2). LCMS; m/z 677 (M + 1). IR: 3069, 3037, 2944, 2883, 1597, 1495, 1451, 1334, 1286, 1257, 1165, 1144, 1117, 1094, 1071, 1056, 951, 928, 866, 817, 797, 750, 721, 665, 579, 549. ${ }^{1} \mathrm{H}$ NMR $\left(\mathrm{CDCl}_{3}\right): \delta 7.62$ (d, 4H, J 7.8), $7.27(\mathrm{t}, 2 \mathrm{H}, J$ 7.8), 7.26 (d, $4 \mathrm{H}, J$ 7.8), 7.19 (d, 2H, J 7.8), $6.93\left(\mathrm{t}, 2 \mathrm{H}, J\right.$ 7.6), $6.82\left(\mathrm{~d}, 2 \mathrm{H}, J\right.$ 8.2), $5.75\left(\mathrm{~m}, 2 \mathrm{H}, \mathrm{CH}=\mathrm{CH}_{2}\right)$, 
5.06 (d, 2H, J 17, $\left.\mathrm{CH}_{2}=\mathrm{CH}\right), 4.97\left(\mathrm{~d}, 2 \mathrm{H}, J 10, \mathrm{CH}_{2}=\mathrm{CH}\right), 4.22$ (d, 4H, J 4.2), 3.86 (br, 4H), $3.51(\mathrm{t}, 4 \mathrm{H}, J 4.3), 2.43\left(\mathrm{~s}, 6 \mathrm{H}, \mathrm{CH}_{3}\right) .{ }^{13} \mathrm{C} \mathrm{NMR}\left(\mathrm{CDCl}_{3}\right): \delta 155.9,142.8,137.7,133.5,132.8$, 129.6, 129.1, 127.6, 126.9, 120.8, 118.2, 112.6, 69.3, 67.3, 52.6, 21.5. Anal. calcd. for $\mathrm{C}_{36} \mathrm{H}_{40} \mathrm{~N}_{2} \mathrm{O}_{7} \mathrm{~S}_{2}$ (676.9): C 63.88; H 5.96; N 4.14; S 9.47\%. Found: C 63.92; H 6.01; N 4.40; S 9.28\%.

Compound 4d. Yield 50\% (A), 83\% (B); brown oil (EtOAc/pet. ether 40-60), $\mathrm{R}_{\mathrm{f}}=0.6$ (EtOAc/pet. ether 40-60, 2:1). LCMS; m/z $721(\mathrm{M}+1)$. IR: 3069, 3025, 2927, 2876, 1597, 1496, 1451, 1344, 1287, 1257, 1163, 1118, 1093, 1061, 929, 815, 754, 712, 665, 578, 551. ${ }^{1} \mathrm{H}$ NMR $\left(\mathrm{CDCl}_{3}\right): \delta 7.59(\mathrm{~d}, 4 \mathrm{H}, J$ 8.1), $7.24(\mathrm{~m}, 8 \mathrm{H}), 6.93(\mathrm{t}, 2 \mathrm{H}, J$ 7.5), $6.78(\mathrm{~d}, 2 \mathrm{H}, J$ 8.1), $5.76(\mathrm{~m}, 2 \mathrm{H}$, $\left.-\mathrm{CH}=\mathrm{CH}_{2}\right), 5.09\left(\mathrm{dd}, 2 \mathrm{H}, J\right.$ 17. 0.7, $\left.-\mathrm{CH}=\mathrm{CH}_{2}\right), 4.98\left(\mathrm{~d}, 2 \mathrm{H}, \mathrm{J} 10.1,-\mathrm{CH}=\mathrm{CH}_{2}\right), 4.23(\mathrm{br}, 4 \mathrm{H})$, $3.82(\mathrm{br}, 4 \mathrm{H}), 3.61(\mathrm{~s}, 4 \mathrm{H}), 3.43(\mathrm{t}, 4 \mathrm{H}, J 4.5), 2.42\left(\mathrm{~s}, 6 \mathrm{H}, \mathrm{CH}_{3}\right) .{ }^{13} \mathrm{C} \mathrm{NMR}\left(\mathrm{CDCl}_{3}\right): \delta 155.6$, 142.8, 137.6, 133.6, 133.2, 129.6, 129.1, 127.6, 126.6, 120.6, 118.2, 112.2, 70.7, 69, 67, 52.4, 21.5. Anal. calcd. for $\mathrm{C}_{38} \mathrm{H}_{44} \mathrm{~N}_{2} \mathrm{O}_{8} \mathrm{~S}_{2}$ (720.9): C 63.31; H 6.15; N 3.89; S 8.90\%. Found: C 62.99; H 6.21; N 4.11; S 8.62\%.

Compound 6d. Yield 56\% (A), 54\% (B); colorless crystals (EtOH), $\mathrm{R}_{\mathrm{f}}=0.9$ (DCM/pet. ether 4060/EtOAc 6:4:2). MS; m/z $932\left(\mathrm{M}^{+}\right)$. IR: 3434, 3073, 2922, 2873, 1599, 1496, 1453, 1341, 1286, 1253, 1161, 1119, 1094, 1044, 754, 658, 573, 554. ${ }^{1} \mathrm{H}$ NMR $\left(\mathrm{CDCl}_{3}\right): \delta 7.61(\mathrm{~d}, 4 \mathrm{H}, J$ 8.0), 7.44 (d, 2H, J 7.4), 7.22 (d, 4H, J 8.0), 7.17-7.07 (m, 6H), 6.84 (t, 2H, J 7.4), 6.78 (t, 2H, J 7.6), 6.70 $\left(\mathrm{d}, 4 \mathrm{H}, J\right.$ 8.2), $5.88\left(\mathrm{~m}, 2 \mathrm{H},-\mathrm{CH}=\mathrm{CH}_{2}\right), 5.30\left(\mathrm{dd}, 2 \mathrm{H}, J 17.3,1.0,-\mathrm{CH}_{2}=\mathrm{CH}\right), 5.19(\mathrm{dd}, 2 \mathrm{H}, J$ $\left.10.8,1.0,-\mathrm{CH}_{2}=\mathrm{CH}\right), 4.88(\mathrm{br}, 4 \mathrm{H}), 4.35(\mathrm{~d}, 4 \mathrm{H}, \mathrm{J} 4.6), 3.76(\mathrm{br} \mathrm{s}, 4 \mathrm{H}), 3.67(\mathrm{~s}, 4 \mathrm{H}), 3.47(\mathrm{t}, 4 \mathrm{H}$, $J$ 5.1), $2.41\left(\mathrm{~s}, 6 \mathrm{H}, \mathrm{CH}_{3}\right) .{ }^{13} \mathrm{C} \mathrm{NMR}\left(\mathrm{CDCl}_{3}\right): \delta 156.4,155.8,142.5,138.0,133.4,133.0,130.7$, 129.3, 129.0, 128.6, 127.7, 127.2, 125.3, 120.4 (two overlapped $\mathrm{CH}$ ), 116.8, 112.0, 111.3, 70.8, 69.1, 68.7, 66.9, 48.3, 21.5. Anal. calcd. for $\mathrm{C}_{52} \mathrm{H}_{56} \mathrm{~N}_{2} \mathrm{O}_{10} \mathrm{~S}_{2}$ (933.1): C 66.93; H 6.05; N 3.00; $\mathrm{S}$ 6.87\%. Found: C 66.22; H 6.01; N 3.19; S 6.34\%.

\section{General procedures for RCM}

To a solution of each of 2a-e, 4c,d, 6 (2 mmol) in DCM $(10 \mathrm{ml})$, Grubbs' catalyst I (1-5\%) was added. The reaction mixture was heated under reflux for $2 \mathrm{~h}$, the solvent was removed in vacuo and the product was crystallized from the appropriate solvent or purified by column chromatography.

Compound 3a. Yield 0.6 g (80\%); colorless crystals (EtOH), mp 127-128 ${ }^{\circ} \mathrm{C}, \mathrm{R}_{\mathrm{f}}=0.6$ (DCM/pet. ether 40-60/EtOAc 6:4:2). LCMS; m/z 693 (M + 1). IR: 3066, 3024, 2926, 2869, 1596, 1496, $1451,1346,1283,1254,1215,1163,1122,1091,1073,816,756,656,577,554 .{ }^{1} \mathrm{H}$ NMR $\left(\mathrm{CDCl}_{3}\right) E$-isomer: $\delta 7.59(\mathrm{~m}, 4 \mathrm{H}), 7.38(\mathrm{~m}, 2 \mathrm{H}), 7.28(\mathrm{~m}, 4 \mathrm{H}), 7.09(\mathrm{~d}, 2 \mathrm{H}, J$ 8.2), 6.94-6.99 (m, $4 \mathrm{H}), 5.26(\mathrm{~s}, 2 \mathrm{H}, \mathrm{CH}=), 3.74(\mathrm{~s}, 4 \mathrm{H}), 3.50-4.20($ br m, 8H), 3.35 (t, 4H, J 6.4), 2.45 (s, 6H, $\left.\mathrm{CH}_{3}\right) .{ }^{13} \mathrm{C} \mathrm{NMR}\left(\mathrm{CDCl}_{3}\right)$ E-isomer: $\delta 157,143.1,136.7,131.1,129.8,129.3,129.2,127.8,127.7$, 121.2, 114.4, 70.6, 68.1, 67.1, 49.9, 21.5. ${ }^{1} \mathrm{H}$ NMR $\left(\mathrm{CDCl}_{3}\right)$ Z-isomer: $\delta 7.59(\mathrm{~m}, 4 \mathrm{H}), 7.38(\mathrm{~m}$, 2H), $7.28(\mathrm{~m}, 4 \mathrm{H}), 7.16(\mathrm{~d}, 2 \mathrm{H}, J$ 8.8), 6.94-6.99 (m, 2H), $6.83(\mathrm{~d}, 2 \mathrm{H}, J$ 8), 5.47 (t, 2H, J 3.6, $\mathrm{CH}=), 3.84(\mathrm{~s}, 4 \mathrm{H}), 3.50-4.20\left(\right.$ br m, 8H), $3.46\left(\mathrm{t}, 4 \mathrm{H}, J\right.$ 6.6), $2.47\left(\mathrm{~s}, 6 \mathrm{H}, \mathrm{CH}_{3}\right) .{ }^{13} \mathrm{C} \mathrm{NMR}$ 
$\left(\mathrm{CDCl}_{3}\right)$ Z-isomer: $\delta 155.9,142.9,137.4,132.3,129.9,129.4,129.3,127.9,127.7,121.3,113.1$, 67.9, 67.0, 65.9, 49.1, 21.5. Anal. calcd. for $\mathrm{C}_{36} \mathrm{H}_{40} \mathrm{~N}_{2} \mathrm{O}_{8} \mathrm{~S}_{2}$ (692.8): C 62.41; H 5.82; N 4.04; S 9.26\%. Found: C 62.21; H 5.76; N 4.27; S 8.96\%.

Compound 3b. Yield 0.6 g (86\%); colorless crystals (EtOH/DCM), mp 242-243 ${ }^{\circ} \mathrm{C}, \mathrm{R}_{\mathrm{f}}=0.6$ (DCM/pet. ether 40-60/EtOAc 6:4:2). LCMS; m/z 707 (M + 1). IR: 3066, 2941, 2851, 1596, 1497, 1453, 1342, 1280, 1256, 1163, 1124, 1092, 1060, 969, 818, 754, 713, 657, 575, 553. ${ }^{1} \mathrm{H}$ NMR $\left(\mathrm{CDCl}_{3}\right)$ E-isomer: $\delta 7.58(\mathrm{~d}, 4 \mathrm{H}, J 8.2), 7.31(\mathrm{dt}, 2 \mathrm{H}, J$ 8.8, 1.4), 7.27 (d, 4H, J 8.2), 7.11 (dd, 2H, J 7.7, 1.3), 6.91 (t, 2H, J 7.5), 6.87 (d, 2H, J 8.3), 4.93 (s, 2H, CH=), 3.80 (br, 4H), 3.68 $\left(\mathrm{t}, 4 \mathrm{H}, J\right.$ 5.7), $3.60(\mathrm{~s}, 4 \mathrm{H}), 3.33(\mathrm{~s}, 4 \mathrm{H}), 2.44\left(\mathrm{~s}, 6 \mathrm{H}, \mathrm{CH}_{3}\right), 1.62(\mathrm{q}, 2 \mathrm{H}, J 5.8) .{ }^{13} \mathrm{C} \mathrm{NMR}\left(\mathrm{CDCl}_{3}\right)$ E-isomer: $\delta 156.5,142.9,136.9,131.9,129.7,129.2,129.1,127.6,126.9,120.6,112.2,70.4,67$, 63.5, 49.5, 28.1, 21.5. Anal. calcd. for $\mathrm{C}_{37} \mathrm{H}_{42} \mathrm{~N}_{2} \mathrm{O}_{8} \mathrm{~S}_{2}$ (706.9): C 62.88; H 5.94; N 3.96; S 9.06\%. Found: C 62.40; H 6.42; N 4.12; S 9.06\%.

Compound 3c. Yield 0.6 g (80\%); colorless crystals (EtOH), mp 129-130 ${ }^{\circ} \mathrm{C}, \mathrm{R}_{\mathrm{f}}=0.4$ (DCM/pet. ether 40-60/EtOAc 6:4:2). LCMS; m/z 737 (M + 1). IR: 3420, 2927, 2867, 1597, 1497, 1449, $1345,1278,1166,1138,1094,1069,936,814,761,711,658,578,553 .{ }^{1} \mathrm{H} \mathrm{NMR}\left(\mathrm{CDCl}_{3}\right) E-$ isomer: $\delta 7.61$ (two overlapped doublets, $4 \mathrm{H}, J$ 8.1), 7.21-7.30 (m, 6H), $7.16(\mathrm{dd}, 2 \mathrm{H}, J 7.7,1.3)$, $6.93(\mathrm{t}, 2 \mathrm{H}, J$ 7.4), 6.86 (d, 2H, J 8.2), 5.28 (br, 2H, $\mathrm{CH}=), 3.89(\mathrm{~s}, 3 \mathrm{H}), 3.77-3.72(\mathrm{~m}, 8 \mathrm{H}), 3.50$ $(\mathrm{s}, 3 \mathrm{H}), 3.42-3.36(\mathrm{~m}, 6 \mathrm{H}), 2.43\left(\mathrm{~s}, 6 \mathrm{H}, \mathrm{CH}_{3}\right) .{ }^{13} \mathrm{C} \mathrm{NMR}\left(\mathrm{CDCl}_{3}\right)$ E-isomer: $\delta$ 156.1, 142.9, 137.1, 132.3, 129.6, 129.4, 129.1, 127.7, 127.7, 120.8, 112.7, 70.4, 68.8, 67.4, 67.3, 49.2, 21.5. ${ }^{1} \mathrm{H}$ NMR $\left(\mathrm{CDCl}_{3}\right)$ Z-isomer: $\delta 7.61$ (two overlapped doublets, $4 \mathrm{H}, J$ 8.1), 7.21-7.30 (m, 8H), 6.93 (t, 2H, J 7.4), 6.81 (d, 2H, J 8.2), 5.28 (br, 2H, CH=), 3.82 (s, 4H), 3.77-3.72 (m, 8H), 3.42$3.36(\mathrm{~m}, 8 \mathrm{H}), 2.44\left(\mathrm{~s}, 6 \mathrm{H}, \mathrm{CH}_{3}\right) .{ }^{13} \mathrm{C} \mathrm{NMR}\left(\mathrm{CDCl}_{3}\right)$ Z-isomer: $\delta$ 155.8, 142.8, 137.4, 132.8, 129.6, 129.2, 129.1, 127.3, 127.1, 120.9, 112.4, 69.1, 67.5, 67.3, 65.3, 48.5, 21.5. Anal. calcd. for $\mathrm{C}_{38} \mathrm{H}_{44} \mathrm{~N}_{2} \mathrm{O}_{9} \mathrm{~S}_{2}$ (736.9): C 61.94; H 6.02; N 3.80; S 8.70\%. Found: C 61.62; H 5.98; N 3.93; S $8.37 \%$.

Compound 3d. Yield $0.47 \mathrm{~g}(60 \%)$; colorless crystals, purified by column chromatography (EtOAc/pet. ether 40-60, 2:1), $\mathrm{mp} 57-58{ }^{\circ} \mathrm{C}, \mathrm{R}_{\mathrm{f}}=0.6$ (EtOAc/pet. ether 40-60, 2:1). LCMS; $\mathrm{m} / \mathrm{z}$ $781(\mathrm{M}+1)$. IR: 3064, 3023, 2926, 2870, 1596, 1496, 1452, 1345, 1286, 1163, 1119, 1072, 755. ${ }^{1} \mathrm{H}$ NMR $\left(\mathrm{CDCl}_{3}\right)$ E-isomer: $\delta 7.59(\mathrm{~m}, 4 \mathrm{H}), 7.19-7.25(\mathrm{~m}, 8 \mathrm{H}), 6.93(\mathrm{~m}, 2 \mathrm{H}), 6.80(\mathrm{~d}, 2 \mathrm{H}, J$ 8.1), 5.52 (br s, 2H, CH=), $3.82\left(\mathrm{~s}, 8 \mathrm{H}, \mathrm{CH}_{2}\right), 3.75$ (t, 4H, J 6.6), $3.58(\mathrm{~s}, 4 \mathrm{H}), 3.48$ (t, 8H, J 6.6), 2.42 $\left(\mathrm{s}, 6 \mathrm{H}, \mathrm{CH}_{3}\right) .{ }^{13} \mathrm{C} \mathrm{NMR}\left(\mathrm{CDCl}_{3}\right)$ E-isomer: $\delta$ 155.5, 142.6, 137.7, 134.5, 133.4, 129.7, 129 , 127.6, 127, 120.8, 117.1, 112.4, 72.2, 70, 69.3, 67.9, 67, 48.9, 21.4. ${ }^{1} \mathrm{H} \mathrm{NMR}\left(\mathrm{CDCl}_{3}\right) \mathrm{Z}$-isomer: $\delta 7.59(\mathrm{~m}, 4 \mathrm{H}), 7.19-7.25(\mathrm{~m}, 8 \mathrm{H}), 6.93(\mathrm{~m}, 2 \mathrm{H}), 6.80(\mathrm{~d}, 2 \mathrm{H}, J$ 8.1), $5.44(\mathrm{t}, 2 \mathrm{H}, J 5.4, \mathrm{CH}=)$, $3.86(\mathrm{~s}, 8 \mathrm{H}), 3.77(\mathrm{~s}, 4 \mathrm{H}), 3.61(\mathrm{~s}, 4 \mathrm{H}), 3.43(\mathrm{~s}, 8 \mathrm{H}), 2.45\left(\mathrm{~s}, 6 \mathrm{H}, \mathrm{CH}_{3}\right)$. Anal. calcd. for $\mathrm{C}_{40} \mathrm{H}_{48} \mathrm{~N}_{2} \mathrm{O}_{10} \mathrm{~S}_{2}$ (780.9): C 61.52; H 6.20; N 3.59; $\mathrm{S} 8.21 \%$. Found: C 61.10; H 6.21; N 3.68; S 7.73\%.

Compound 3e. Yield 0.7 g (95\%); purified by column chromatography (DCM/pet. ether 4060/EtOAc 6:4:2), colorless crystals $(\mathrm{EtOH}), \mathrm{mp} 100-101{ }^{\circ} \mathrm{C}, \mathrm{R}_{\mathrm{f}}=0.6(\mathrm{DCM} /$ pet. ether 4060/EtOAc). LCMS; m/z 769 (M + 1). IR: 3064, 3027, 2923, 2857, 1595, 1496, 1452, 1344, 1162 , $1091,1072,1001,756 .{ }^{1} \mathrm{H} \mathrm{NMR}\left(\mathrm{CDCl}_{3}\right) E$ and $Z$ isomers: $\delta$ 7.61-7.57 (m, $\left.4 \mathrm{H}, E, Z\right), 7.26-7.36$ 
(m, 6H, E, Z), 7.17-7.13 (m, 6H, E, Z), 6.96-6.93 (m, 4H, E, Z), 5.35 (t, 2H, J 3.6, CH=, Z), 5.14 (s, 2H, CH=, E), 5.14-5.02 (br, 4H, $\left.\mathrm{CH}_{2}, E, Z\right), 3.65$ (m, 8H, E, Z), 3.44-3.39 (m, 4H, E, Z), 2.38 (s, 6H, $\mathrm{CH}_{3}, E, Z$ ). Anal. calcd. for $\mathrm{C}_{42} \mathrm{H}_{44} \mathrm{~N}_{2} \mathrm{O}_{8} \mathrm{~S}_{2}$ (768.9): C 65.64; H 5.77; N 3.64; S 8.34\%. Found: C 64.88; H 5.77; N 3.86; S 7.97\%.

Compound 5c. Yield $0.49 \mathrm{~g}$ (75\%); colorless crystals (EtOAc/pet. ether 40-60), mp 220-221 ${ }^{\circ} \mathrm{C}$, $\mathrm{R}_{\mathrm{f}}=0.6$ (DCM/pet. ether 40-60/EtOAc 6:4:2). MS; m/z 648. IR: 3024, 2920, 2872, 1597, 1496, 1451, 1343, 1286, 1259, 1160, 1122, 1092, 1063, 753, 578. ${ }^{1} \mathrm{H} \mathrm{NMR}\left(\mathrm{CDCl}_{3}\right)$ E-isomer: $\delta 7.52$ $(\mathrm{m}, 4 \mathrm{H}), 7.31-7.20(\mathrm{~m}, 8 \mathrm{H}), 6.95(\mathrm{t}, 2 \mathrm{H}, J$ 7.4), $5.41(\mathrm{t}, 2 \mathrm{H}, J$ 5, CH=), 3.1-4.1 (m, 12H), $2.42(\mathrm{~s}$, $\left.6 \mathrm{H}, \mathrm{CH}_{3}\right) .{ }^{13} \mathrm{C} \mathrm{NMR}\left(\mathrm{CDCl}_{3}\right)$ E-isomer: $\delta 156,142.5,137.5,132.4,129.5,128.9(2 \mathrm{C}), 127.6$, 126.6, 120.8, 111.7, 68.7, 66.8, 51.4, 21.4. ${ }^{1} \mathrm{H}$ NMR $\left(\mathrm{CDCl}_{3}\right) Z$-isomer: $\delta 7.52(\mathrm{~m}, 4 \mathrm{H}), 7.31-$ $7.20(\mathrm{~m}, 8 \mathrm{H}), 7.08$ (d, 2H, J 7.6), 6.88 (t, 2H,J 8.3), 6.74 (d, 2H, J 8.1), 5.58 (br s, 2H, CH=), 3.1-4.1 (m, 12H), $2.43\left(\mathrm{~s}, 6 \mathrm{H}, \mathrm{CH}_{3}\right) .{ }^{13} \mathrm{C} \mathrm{NMR}\left(\mathrm{CDCl}_{3}\right) \mathrm{Z}$-isomer: $\delta$ 155.3, 142.6, 138.2, 134.6, 129.7, 129.5, 128.9, 127.7, 120.6, 111.6, 67.8, 66.2, 44.3, 21.5. Anal. calcd. for $\mathrm{C}_{34} \mathrm{H}_{36} \mathrm{~N}_{2} \mathrm{O}_{7} \mathrm{~S}_{2}$ (648.8): C 62.94; H 5.59; N 4.32; S 9.88\%. Found: C 62.88; H 5.61; N 4.52; S 9.63\%.

Compound 5d. Yield $0.6 \mathrm{~g}$ (85\%); colorless crystals (EtOH), mp 184-185 ${ }^{\circ} \mathrm{C}, \mathrm{R}_{\mathrm{f}}=0.4$ (DCM/pet. ether 40-60/EtOAc 6:4:2). MS; m/z 692. IR: 3066, 3025, 2926, 2873, 1597, 1496, 1451, 1343, $1286,1259,1216,1161,1122,1092,1064,937,888,814,751,693,658,579,553 .{ }^{1} \mathrm{H}$ NMR $\left(\mathrm{CDCl}_{3}\right)$ E-isomer: $\delta 7.53(\mathrm{~d}, 4 \mathrm{H}, J$ 8.1), 7.21-7.26 (m, 6H), $7.00(\mathrm{dd}, 2 \mathrm{H}, J$ 7.6, 1.1), $6.86(\mathrm{t}, 2 \mathrm{H}$, $J$ 7.5), $6.72\left(\mathrm{~d}, 2 \mathrm{H}, J\right.$ 8.2), $5.41(\mathrm{~s}, 2 \mathrm{H}, \mathrm{CH}=), 3.48-4.20(\mathrm{~m}, 16 \mathrm{H}), 2.42\left(\mathrm{~s}, 6 \mathrm{H}, \mathrm{CH}_{3}\right) .{ }^{13} \mathrm{C} \mathrm{NMR}$ $\left(\mathrm{CDCl}_{3}\right) E$-isomer: $\delta 155.9,142.5,137.3,131.8,129.3,129.2,128.9$ (2C), 127.5, 120.3, 111.6, 70.4, 69, 66.7, 51.6, 21.3. ${ }^{1} \mathrm{H}$ NMR $\left(\mathrm{CDCl}_{3}\right)$ Z-isomer: $\delta 7.53(\mathrm{~d}, 4 \mathrm{H}, J$ 8.1), 7.21-7.26 (m, 6H), $7.00(\mathrm{dd}, 2 \mathrm{H}, J$ 7.6, 1.1), $6.91(\mathrm{~m}, 2 \mathrm{H}), 6.76(\mathrm{~d}, 2 \mathrm{H}, J$ 8.3), 5.35 (s, 2H, CH=), 3.48-4.20 (m, $16 \mathrm{H}), 2.42\left(\mathrm{~s}, 6 \mathrm{H}, \mathrm{CH}_{3}\right) .{ }^{13} \mathrm{C} \mathrm{NMR}\left(\mathrm{CDCl}_{3}\right)$ Z-isomer: $\delta 155.3,142.4,138.1,133.2,129.5,128.3$, 127.4, 126.5, 126.4, 120.5, 112.0, 70.7, 68.8, 67.4, 46.4, 21.3. Anal. calcd. for $\mathrm{C}_{36} \mathrm{H}_{40} \mathrm{~N}_{2} \mathrm{O}_{8} \mathrm{~S}_{2}$ (692.8): C 62.41; H 5.82; N 4.04; S 9.26\%. Found: C 62.24; H 5.86; N 4.25; S 8.62\%.

Compound 7d. Yield $0.7 \mathrm{~g}(82 \%)$; colorless crystals (EtOAc/ pet. ether 40-60), mp 192-193 ${ }^{\circ} \mathrm{C}$, $\mathrm{R}_{\mathrm{f}}=0.7(\mathrm{DCM} /$ pet. ether 40-60/EtOAc 6:4:2). LCMS; m/z $905(\mathrm{M}+1)$. IR: 3066, 3027, 2926, $2872,1599,1495,1453,1342,1286,1256,1238,1220,1160,1120,1092,753 .{ }^{1} \mathrm{H}$ NMR $\left(\mathrm{CDCl}_{3}\right) E$-isomer: $\delta 7.61(\mathrm{~d}, 6 \mathrm{H}, J$ 8.2), 7.23-7.08 (m, 10H), $6.98(\mathrm{t}, 2 \mathrm{H}, J$ 7.4), $6.82(\mathrm{t}, 2 \mathrm{H}, J$ 7.5), 6.71 (d, 2H, J 8.1), 6.62 (d, 2H, J 8.1), 5.70 (s, 2H, CH=), 4.92 (s, 4H), $4.26(\mathrm{~s}, 4 \mathrm{H}), 3.68$ (br, 4H), $3.58(\mathrm{~s}, 4 \mathrm{H}), 3.34(\mathrm{br}, 4 \mathrm{H}), 2.41\left(\mathrm{~s}, 6 \mathrm{H}, \mathrm{CH}_{3}\right) .{ }^{1} \mathrm{H}$ NMR $\left(\mathrm{CDCl}_{3}\right) \mathrm{Z}$-isomer: $\delta 7.62(\mathrm{~d}$, $6 \mathrm{H}, J$ 8.2), 7.23-7.08 (m, 10H), 6.98 (t, 2H, J 7.4), $6.82(\mathrm{t}, 2 \mathrm{H}, J$ 7.5), 6.75 (d, 2H, J 8.3), 6.66 $(\mathrm{m}, 2 \mathrm{H}), 5.65$ (s, 2H, CH=, J 4.4), 4.89 (s, 4H), 4.33 (s, 4H), 3.68 (br, 4H), 3.59 (s, 4H), 3.34 (br, $4 \mathrm{H}), 2.43\left(\mathrm{~s}, 6 \mathrm{H}, \mathrm{CH}_{3}\right) .{ }^{13} \mathrm{C} \mathrm{NMR}\left(\mathrm{CDCl}_{3}\right)$ E-isomer: $\delta 156.3,155.4,142.6,138.2,133.9,130.2$, 129.4, 129, 129, 128.4, 127.7 (2C), 126.5, 121.1, 120.5, 111.9, 111.8, 70.7, 68.9, 68.1, 67.1, 47.7, 21.5. Anal. calcd. for $\mathrm{C}_{50} \mathrm{H}_{52} \mathrm{~N}_{2} \mathrm{O}_{10} \mathrm{~S}_{2}$ (905.1): C 66.35; H 5.79; N 3.10; S 7.09\%. Found: C $66.00 ; \mathrm{H} 5.65 ; \mathrm{N} 3.08 ;$ S $6.86 \%$. 


\section{Acknowledgements}

The support of the University of Kuwait received through research grant no. SC10/02 and the General Facilities Projects grants no. GS01/01 and GS03/01 are gratefully acknowledged.

\section{References}

1. a) Grubbs, R. H. Tetrahedron 2004, 60, 7117. b) Deiters, A.; Martin, S. Chem. Rev. 2004, 104, 2199. c) Nakamura, I.; Yamamoto, Y. Chem. Rev. 2004, 104, 2127. d) Fürstner, A. Angew. Chem. Int. Ed. 2000, 39, 3012. e) Fürstner, A. Ackermann, L. Chem. Commun. 1999, 95, and references cited therein. f) Review: Grubbs, R. H.; Chang, S. Tetrahedron 1998, 54, 4413. g) Fürstner, A. Top. Catal. 1997, 4, 285. h) Ivin, K. J., Mol, J. C. 1997 "Olefin Metathesis and Metathesis Polymerization" Acad. Press, New York.

2. a) Ibrahim, Y. A.; Behbehani, H.; Ibrahim, M. R. Tetrahedron Lett. 2002, 43, 4207. b) Behbehani, H.; Ibrahim, M. R.; Ibrahim, Y. A. Tetrahedron Lett. 2002, 43, 6421. c) Ibrahim, Y. A.; Behbehani, H.; Ibrahim, M. R.; Malhas, R. N. Tetrahedron 2003, 59, 7273. d) Ibrahim, Y. A.; Behbehani, H.; Ibrahim, M. R.; Abrar, N. M. Tetrahedron Lett. 2002, 43, 6971. e) Ibrahim, Y. A.; Behbehani, H.; Khalil, N. S. Tetrahedron 2004, 60, 8429.

3. Malhas, R. N.; Ibrahim, Y. A. Synthesis 2006, 3261.

4. Review: Ibrahim, Y. A. J. Mol. Catal. A: Chemical 2006, 254, 43.

5. Cade, J. F. J. Med. J. Aus. 1949, 36, 349.

6. Paquette, L. A.; Tae, J. J. Am. Chem. Soc. 2001, 123, 4974.

7. Kaplan, A.; Szabo, L. “Clinical Chemistry: Interpretation and Techniques”, Lea and Febiger, Philadelphia, PA (1979).

8. Zhukov, F.; Amman, D.; Guggi, M.; Simon, W. Anal. Chem. Acta. 1981, 131, 117.

9. Metzger, E.; Amman, D.; Asper, R.; Simon, W. Anal. Chem. 1986, 58, 132.

10. Shanzer, A.; Samuel, D.; Korenstein, R. J. Am. Chem. Soc. 1983, 105, 3815.

11. Gadzekpo, P. Y.; Hungerford, J.; Kardry, A.; Ibrahim, Y. A.; Christian, G. Anal. Chem. 1985, $57,493$.

12. Metzger, E.; Dohner, R.; Simon, W. Anal. Chem. 1987, 59, 1600.

13. Gadzekpo, P. Y.; Hungerford, J.; Kardry, A.; Ibrahim, Y. A.; Christian, G. Anal. Chem. 1986, 58, 1948.

14. Umezawa, A. Y.; Umezawa, K.; Ato, S. H. Pure Appl. Chem. 1995, 67, 507.

15 a) Ibrahim, Y. A.; Elwahy, A. H. M.; Abbas, A. A. Tetrahedron 1994, 50, 11489 and references cited therein. b) Elwahy, A. H. M.; Abbas, A. A.; Ibrahim, Y. A. J. Chem. Res. (S) 1998, 184, (M) 1998, 901 and references cited therein.

16 Biernat, J. F.; Luboch, E Tetrahedron 1984, 40, 1927.

17 Kleinpeter, E.; Gaebler, M.; Schorth, W. Magn. Reson. Chem. 1988, 26, 380. 
18 Guemues, G.; Oeztuerk, Z. Z.; Ahsan, V.; Guel, A.; Bekaroglu, O. J. Chem. Soc. Dalton Trans. 1992, 2485.

19 Shoukry, A. F.; Shuaib, N. M.; Ibrahim, Y. A.; Malhas, R. N. Electroanalysis 2005, 17, 713. 